\title{
Investigation of blood flow in the external carotid artery and its branches with a new $0 \mathrm{D}$ peripheral model
}

\author{
Yoshihito Ohhara' ${ }^{1}$, Marie Oshima ${ }^{2,3}$, Toshinori Iwai ${ }^{*}$, Hiroaki Kitajima', Yasuharu Yajima' ${ }^{1}$, Kenji Mitsudo',
} Absy Krdy ${ }^{2,3}$ and Iwai Tohnai ${ }^{1}$

\section{*Correspondence:}

iwai104oams@yahoo.co.jp

${ }^{1}$ Department of Oral

and Maxillofacial Surgery,

Yokohama City University

Graduate School of Medicine,

3-9 Fukuura, Kanazawa-ku, Yokohama 236-0004, Japan

Full list of author information is available at the end of the article

\begin{abstract}
Background: Patient-specific modelling in clinical studies requires a realistic simulation to be performed within a reasonable computational time. The aim of this study was to develop simple but realistic outflow boundary conditions for patient-specific blood flow simulation which can be used to clarify the distribution of the anticancer agent in intra-arterial chemotherapy for oral cancer.

Methods: In this study, the boundary conditions are expressed as a zero dimension (OD) resistance model of the peripheral vessel network based on the fractal characteristics of branching arteries combined with knowledge of the circulatory system and the energy minimization principle. This resistance model was applied to four patient-specific blood flow simulations at the region where the common carotid artery bifurcates into the internal and external carotid arteries.
\end{abstract}

Results: Results of these simulations with the proposed boundary conditions were compared with the results of ultrasound measurements for the same patients. The pressure was found to be within the physiological range. The difference in velocity in the superficial temporal artery results in an error of $5.21 \pm 0.78 \%$ between the numerical results and the measurement data.

Conclusions: The proposed outflow boundary conditions, therefore, constitute a simple resistance-based model and can be used for performing accurate simulations with commercial fluid dynamics software.

Keywords: Blood flow, External carotid artery and its branches, OD peripheral model, Computational fluid dynamics, Simulation, Oral cancer, Intra-arterial chemotherapy

\section{Background}

The standard treatment for oral cancer is surgery, but radical surgery for advanced oral cancer often causes severe oral dysfunction, including speech and swallowing disorders. However, organ and function preservation can be maximized by adopting a multidisciplinary approach that combines radiotherapy and chemotherapy [1]. Selective or superselective intra-arterial chemotherapy, in particular, plays an important role in obviating the need for radical surgery. However, the side effects can be severe and the distribution of the anticancer agent to tumour-feeding arteries is unclear. Therefore, it

(c) 2016 Ohhara et al. This article is distributed under the terms of the Creative Commons Attribution 4.0 International License (http://creativecommons.org/licenses/by/4.0/), which permits unrestricted use, distribution, and reproduction in any medium, provided you give appropriate credit to the original author(s) and the source, provide a link to the Creative Commons license, and indicate if changes were made. The Creative Commons Public Domain Dedication waiver (http://creativecommons.org/publicdomain/zero/1.0/) applies to the data made available in this article, unless otherwise stated. 
is necessary to clarify the optimal dose of anticancer agent for each patient, and it is critical to obtain accurate information on flow distribution in each vessel for optimal drug delivery. Even though the final target of this study is to understand the distribution of the anticancer agent in the carotid artery area, including the branches of the external carotid artery (ECA) which act as feeding arteries for oral cancer, the present study focuses on patient-specific blood flow simulations in the area relevant for intra-arterial chemotherapy since anticancer agent flows are correlated with the blood flow due to their small concentration. Despite the recent progress of measurement methods, such as ultrasound-based methods, it is still difficult to obtain accurate information about the flow distribution in vessels, such as branches of the ECA, in deep soft tissues.

Due to remarkable progress over the last two decades, computational methods have allowed us to conduct realistic haemodynamic simulations. Simulations have also recently been performed in the fields of neurosurgery and cardiovascular surgery for atherosclerosis and aneurysm [2-18]. Simulation is also used as a preoperative examination tool [19-23]. More recent patient-specific 3D model studies have been oriented to the carotid haemodynamics in order to establish correlation between blood flowinduced wall shear stress and atherosclerosis using patient-specific carotid models [24-29]. Using patient-specific internal carotid artery (ICA) model, mechanical stresses also has been analysed by blood flow simulation [30]. In this regard, simple and versatile computational haemodynamic simulation using patient-specific imaging data before treatment is expected to improve treatment quality and allow surgeons to predict treatment results. Also, by using patient-specific imaging data, the postoperative outcome can be compared with the preoperative state. However, it is difficult for surgeons to conduct numerical simulations since they require a certain set of skills related to computers and numerical algorithms, which are outside the scope of clinical practice. Therefore, the use of simulation as a tool to assist treatment can be very challenging. Furthermore, the simulation must yield accurate results within a reasonable time in order for it to be applicable to individual patients.

To achieve a more realistic simulation, the boundary conditions should include the effects of the entire circulatory system. Although a number of numerical models for these boundary conditions have been developed, most models, such as the Windkessel model [31-33] and the lumped parameter model [34-36], rely on physiological parameters, such as resistance, compliance and conductance, which can be determined only from measurement data. Another approach is to conduct a multi-scale simulation combined with a one dimensional-zero dimensional (1D-0D) simulation including the entire circulatory system developed by Liang et al. [37]. Although the multi-scale simulation with the entire circulatory system is more physiologically accurate, it is complicated and time consuming. Since our region of interest is limited to the head and neck area, it is not necessary to conduct a simulation of the entire circulatory system. However, it is necessary to consider the influence of the peripheral blood vessels. Therefore, in this study, we develop a systematic method to determine physiological parameters, such as the resistance of the peripheral network, based on anatomical and physiological knowledge and model the boundary conditions with a 0D model, in order to simulate the effects of the entire circulatory system. The $0 \mathrm{D}$ resistance model of vessels is created in such a way that the peripheral vessel network is constructed as a binary symmetric 
tree that is attached to the outlets of a three-dimensional (3D) geometric structure. As a result, the resistance model defines a relationship between pressure and flow at the outlets such as the outflow boundary conditions in the 3D blood flow simulation. Since the peripheral vessel network of the ECA consists of smaller blood vessels, they tend to be mechanically stiff so the model takes into account changes in the ratio of vessel length to diameter, obtained either from medical imaging data or from anatomical knowledge of smaller vessels. The proposed model also considers non-Newtonian effects in blood viscosity with respect to changes in vessel diameter by using the constitutive relation between the apparent viscosity of blood and the haematocrit.

Because it is more convenient to use commercial software rather than custom-developed software in clinical studies, we have developed a simple boundary condition which can be implemented in commercial software. The model was applied to two patients in order to investigate the flow rate in branching arteries over a large number of cardiac cycles. A comparison between the numerical simulations and the ultrasound measurement data of the flow rates in the superficial temporal artery (STA) of the two patients was performed.

\section{Methods}

This study was reviewed and approved by the Ethics Committee of Yokohama City University (No. B110512003). Written informed consent to use image data was obtained from oral cancer patients.

\section{Patient-specific geometric model}

\section{Computed tomography angiography}

Patients with oral cancer underwent computed tomography (CT) angiography before intra-arterial chemotherapy. We used a 64-slice CT scanner (Aquilion 64; Toshiba Medical Systems, Tokyo, Japan), and non-ionic contrast medium (100 ml) was injected at a rate of $4.0 \mathrm{ml} / \mathrm{s}$ through an antecubital vein with an automatic power injector. A bolus-tracking technique was used to select the individual scan delay of the arterial phase. Repetitive low-dose scans were performed with a delay of $8 \mathrm{~s}$ at a level inferior to the carotid bifurcation. To measure the bolus arrival time, the region of interest was chosen to lie in the common carotid artery (CCA). As soon as an enhancement level of 90 Hounsfield units was reached, the scanning procedure started automatically. For the arterial phase scan, the scanning volume included the inferior margin of the thyroid cartilage/bottom of $\mathrm{C} 6$ and the superior margin of the orbit. The scanner settings were $120 \mathrm{kV}, 250 \mathrm{~mA}, 64 \times 0.5 \mathrm{~mm}$ slice collimation, table speed $20.5 \mathrm{~mm} /$ rotation (pitch 0.641 ), and rotation time $0.75 \mathrm{~s}$. The resolution matrix was $512 \times 512$ pixels with a slice thickness of $1 \mathrm{~mm}$.

\section{Patient-specific model}

Digital imaging and communication in medicine (DICOM) data from the CT angiography of the two patients (right lower gingival cancer and right tongue cancer) were input into Mimics software (Materialise, Leuven, Belgium). The bilateral carotid arteries of each patient were segmented, and thus four separate three-dimensional patient-specific analysis models were created (Fig. 1a, b). The models included the CCA, the ICA, the 


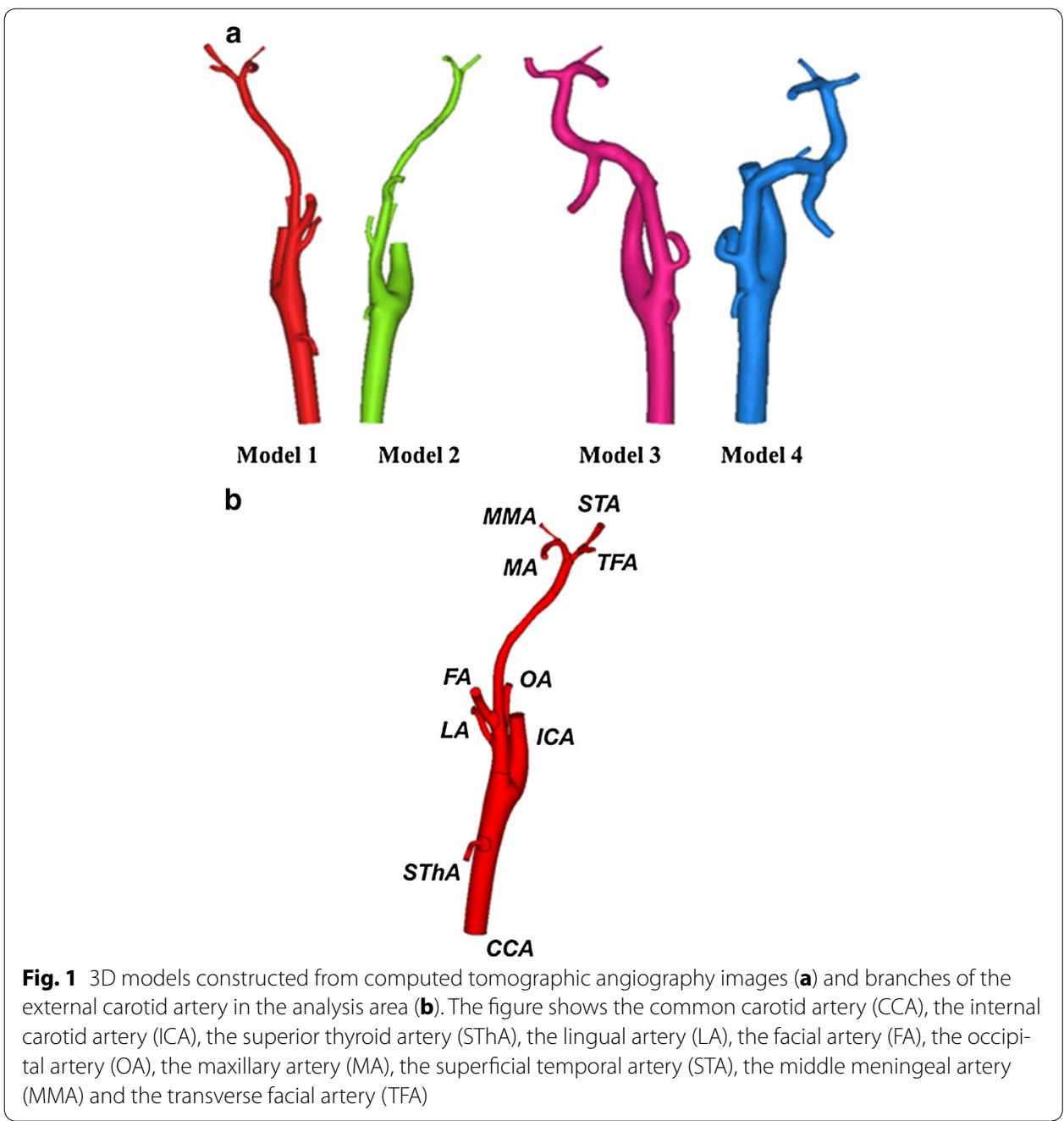

ECA and ECA branches, such as the superior thyroid artery (SThA), lingual artery (LA), facial artery (FA), occipital artery (OA), maxillary artery (MA), posterior auricular artery (PAA), STA, middle meningeal artery (MMA) and transverse facial artery (TFA). Due to limitations in CT resolution, models 1 and 2 included no PAA, models 2-4 had no TFA, and model 2 had no MMA. Therefore, models 1, 3 and 4 had nine branches, and model 2 had seven branches.

\section{Mesh generation}

Before mesh generation for a simulation, the diameters of each artery immediately after branching were measured. In addition, each artery was cut off at a length 5 times the diameter in order to minimize the influences of both inflow and outflow boundary conditions on the flow distributions (Fig. 2a). Numerical analyses with the finite volume method (FVM) were performed using the general-purpose fluid dynamics software FLUENT (Ansys Inc, Canonsburg, PA). The meshes for the FVM were generated using ICEM-CFD software (Ansys Inc., Canonsburg, PA). The mesh consists of tetrahedral cells in the artery core and prismatic cells in the region near the artery wall, as shown in Fig. 2a, and the total number of cells was approximately 3,000,000. Due to this mesh 


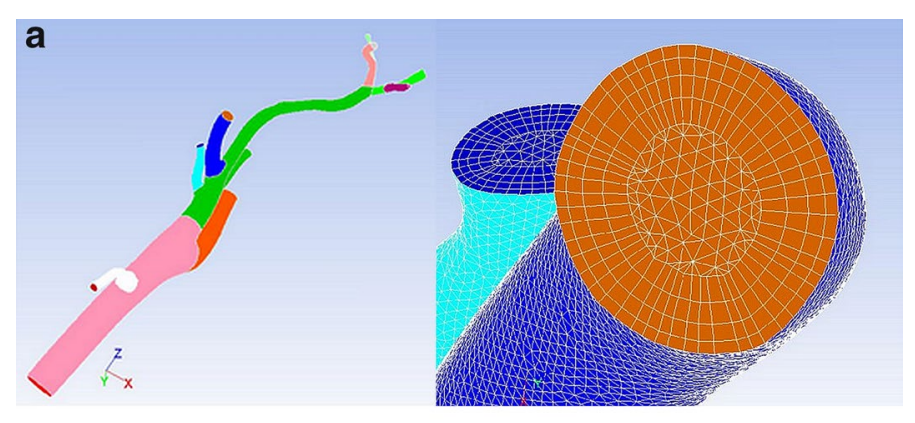

b

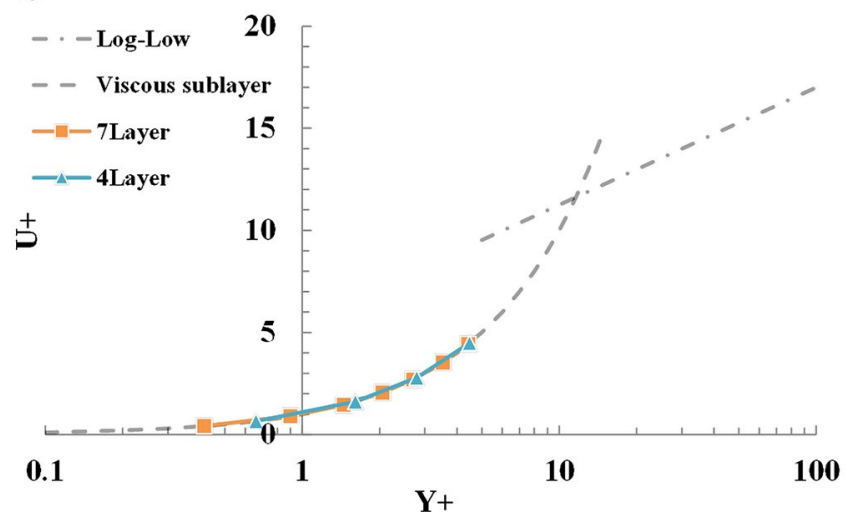

Fig. 2 Tetrahedral mesh with a prismatic boundary layer (a) and mesh value of $y+$ less than one in each vessel (b)

configuration, the cells in the core region are regular while orthogonality was maintained near the wall. In order to resolve the boundary layer, it is necessary to create a fine mesh near the wall, but the calculation time results in increase. Because we suppose to use as a future clinical tool, the mesh requires to minimize within a range that does not affect the present analysis. Therefore, we adopted a tetra prism type mesh. In this paper, the first grid point from the wall was located within $y^{+}<1$, as shown in Fig. $2 b$. There were four layers of prismatic cells in the viscous layer, and the mesh resolution was good enough for the analysis.

\section{Outflow boundary conditions}

We performed 3D blood flow simulations using three types of outflow boundary conditions as the alternative boundary conditions for patient-specific vessels models to be described later in detail: (1) zero-pressure outflow boundary conditions, (2) the pressure boundary conditions with the conventional OD resistance model and (3) the pressure boundary conditions with the present $0 \mathrm{D}$ resistance model. In both $0 \mathrm{D}$ resistance models, a capillary with a radius of $12 \mu \mathrm{m}$ or less was set to be the end of a branch. The conventional model used a constant $\lambda$ (the ratio of the vessel length to its radius of 30) while $\lambda$ in the present model varied depending on a size of a blood vessel diameter. Also, the terminal resistance in the present model was adjusted so that the terminal pressure became $30 \mathrm{mmHg}$, which is within the physiological range of the blood pressure. In two types of $0 \mathrm{D}$ resistance model, the flow rates acquired from the $3 \mathrm{D}$ analysis became the 
inflow boundary condtions for the $\mathrm{OD}$ analysis while the pressure from the $\mathrm{OD}$ analysis became the outflow boundary conditons for the 3D analysis. For the zero-pressure boundary condition, the pressures in all outlets were fixed at zero pressure in all outlets.

\section{Numerical methods}

Since the 3D simulation was conducted by regarding blood as an incompressible Newtonian fluid $\left(1050 \mathrm{~kg} / \mathrm{m}^{3}\right.$ density and $0.0046 \mathrm{~Pa} \cdot \mathrm{s}$ viscosity) in laminar flow with the average properties of blood, blood vessel walls can be regarded as rigid and the no-slip condition can be applied. Inflow boundary conditions of a constant blood flow velocity (Ultrasound system) were prescribed. Since the time scale of administration of drugs in intra-arterial chemotherapy is at least $1 \mathrm{~h}$, a steady state blood simulation is performed instead of an unsteady pulsatile simulation. Three types of outflow boundary conditions for pressure on the outlets (OD resistance models) were prescribed.

The finite volume commercial software Ansys FLUENT was used to carry out steady states fluid dynamics simulations. A SIMPLE scheme was used to couple velocity and pressure and the discretization was accurate to the second order. The 0D resistance models are used as input data for user-defined functions in the code.

Simulations were performed on a PC running the Microsoft Windows 7 Professional operating system. The CPU was a dual-core Intel Xeon W5590 (clock frequency: $3.33 \mathrm{GHz}$ ) and there was $64 \mathrm{~GB}$ of RAM per core. The convergence of the simulations was verified by monitoring the mass flow rate of each outlet and the three velocity components. Convergence was achieved when each of these monitored values and the residuals showed oscillations of the order of $10^{-4}$ or less around a constant mean value. Most of the simulations required a total wall-clock time of less than $26 \mathrm{~h}$.

\section{Mathematical modelling of outflow boundary conditions \\ Physiological background}

In order to achieve a realistic blood flow simulation, it is important to model appropriate physiological conditions, particularly boundary conditions. Even though the systemic arteries are compliant, vessels become stiffer with a smaller radius. Blood vessels are organized in a bifurcating tree in which the total cross-sectional area of the vessels expands from approximately $5 \mathrm{~cm}^{2}$ at the aortic root to approximately $400 \mathrm{~cm}^{2}$ at the arterioles [38]. This expansion in the total cross-sectional area occurs despite the decrease in diameter of individual vessels because the number of daughter vessels doubles at each bifurcation, as shown in Fig. 3. As the number of bifurcations increases, the flow resistance becomes higher and the pressure falls as blood flows through the arterial tree. In this paper, we adopt outflow boundary conditions based on a $0 \mathrm{D}$ resistance model to reflect the effects of the peripheral network on the 3D blood flow simulation; this allows us to conduct simulations using commercial software within a reasonable computation time. Since the diameter of the outlet in the 3D model varies, our OD resistance model is divided into six groups (aorta, large arteries, main artery branches, terminal artery branches, arterioles and capillaries) depending on vessel diameter, as summarized in Fig. 4. 
Fig. 3 A parent vessel splitting into two daughter vessels
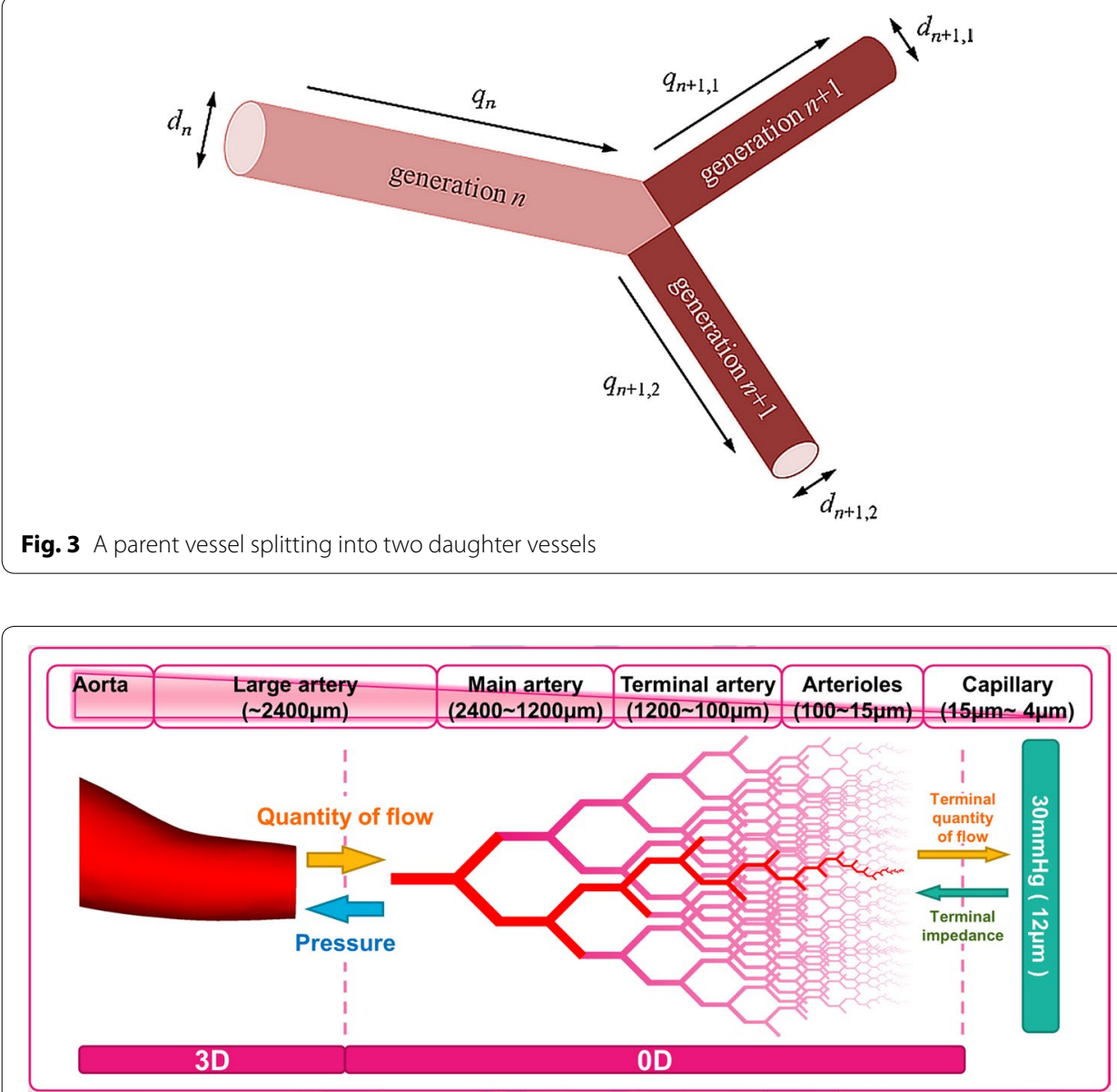

Fig. 4 Anatomical components in the OD resistance model

\section{Geometrical modelling of the peripheral vessel network}

The resolution of CT or magnetic resonance (MR) imaging is not sufficiently high for accurate geometric modelling of a peripheral blood vessel network with small arteries, arterioles and capillaries. Instead, the peripheral network is constructed with a self-similar geometry. The parent blood vessel of generation $i$ has a diameter $d_{i}$, and the flow rate $q_{i}$ is divided into two branches in two daughter generations $(i+1,1)$ and $(i+1,2)$, as shown in Fig. 3. The geometry of peripheral blood vessels network of the OD model is built on the basis of fractal due to anatomical knowledge [39]. In this study, we assume that the flow rate is distributed equally between the two symmetrical daughter vessels [40]:

$$
\begin{aligned}
& q_{i}=q_{i+1,1}+q_{i+1,2} \\
& q_{i+1,1}=q_{i+1,2}=0.5 q_{i}, \quad i=1, \ldots, n
\end{aligned}
$$

where $n$ denotes the total number of generations.

The diameters of the daughter generations are determined in accordance with a power law. This relation was suggested by Uylings [41] and was derived on the basis of the principle of minimum energy in the arterial system: 


$$
\begin{aligned}
& d_{i}^{\varepsilon}=d_{i+1,1}^{\varepsilon}+d_{i+1,2}^{\varepsilon} \\
& d_{i+1,1}^{\varepsilon}=d_{i+1,2}^{\varepsilon}=0.5 d_{i}^{\varepsilon}
\end{aligned}
$$

where $\varepsilon=3.0$ for laminar flow and $\varepsilon=2.33$ for turbulent flow. In this study, $\varepsilon$ was set to 3.0 because the Reynolds number was smaller than 1000 .

\section{OD resistance model}

The outflow boundary conditions were developed as a $0 \mathrm{D}$ model to represent only the resistance of smaller arteries in the peripheral vessel network because this is easy to implement in the software used for the flow simulation. Since the peripheral network consists of relatively small blood vessels, the influence of resistance can be assumed to be more pronounced than that of compliance [42]. However, the elasticity of the arterioles and capillaries was reduced to reflect their properties.

Since the length of small arteries cannot be determined by medical imaging, many authors have discussed methods for determining the length of vessels. For example, Olufsen et al. [43] considered the ratio of vessel length to vessel radius $\lambda=l / r$ and set the ratio $\lambda$ to $50 \pm 10$ [44-46]. In the $0 \mathrm{D}$ models developed by Olufsen et al. [43], it was possible to produce a physiological pressure drop, but it was not possible to calculate the physiological pressure. Therefore, in this research, to calculate the physiological pressure, a pressure of $30 \mathrm{mmHg}$ was applied to the capillary vessel domain at the end of the conventional 0D models. In the basic experiment, we calculated the applied physiological pressure to the peripheral vessel with $\lambda$ fixed at $20,30,40$ or 50 . As a result, the average outlet pressure in $3 \mathrm{D}$ became $75,88,100$, or $122 \mathrm{mmHg}$. The average physiological pressure from arteries to arterioles would be too high at $100 \mathrm{mmHg}$, and excessively low at $75 \mathrm{mmHg}$. Therefore, in the conventional $0 \mathrm{D}$ resistance model used for comparison in this research, the value of $\lambda$ was set to 30 , and terminal resistance values were set to 0 at the end of the network of blood vessels.

We can write the relationship between pressure drop and flow rate in the time domain as follows:

$$
\frac{P_{n}(L)}{Q_{n}(L)}=R_{n}(L)
$$

where $P, Q$ and $R$ denote pressure, quantity of flow and resistance, respectively, and the suffix $n$ denotes the generation of bifurcation. The present resistance model involves two steps, as described above.

The first step is to calculate the resistance $R_{i}(0)$ at the inlet of the artery at the $i$-th generation:

$$
R_{i}(0)=\frac{8 \mu \lambda}{\left(\pi r_{i}^{3}\right)}+R_{i}(L)
$$

where $\mu$ is the viscosity of blood, $\lambda$ is the ratio of the vessel length to its radius, and $R_{i}(L)$ is the resistance at outlet at the $i$-th generation, in which $L$ is the distance from the inlet to the outlet at the $i$-th generation. 
At a bifurcation point in the peripheral network, the resistance follows the same logic as resistance in electric circuits. If we assume that the parent vessel bifurcates into two daughter vessels symmetrically, the relation between the resistances of the parent and daughter vessels is given by

$$
\frac{1}{R_{i}(L)}=\frac{1}{R_{i+1,1}(0)}+\frac{1}{R_{i+1,2}(0)}
$$

The second step is to compute the resistance $R_{i}(L)$ at the outlet of the artery at the $i$-th generation:

$$
R_{i}(L)=\frac{1}{2} R_{i+1,1}(0)
$$

We categorized the vessels in the peripheral network into six groups based on their diameter and length, in accordance with a previous study [47], and the average value of $\lambda$ for each group was determined. If the radius of the artery at the $i$-th generation indicates that the vessel is in the $k$-th group, $\lambda_{i}$ is given by the value of $\lambda$ for the $k$-th group shown in Table 1.

The advantage of the $0 \mathrm{D}$ resistance model is its flexibility in terms of setting the terminal resistance to a value in the physiological range, which is impossible with the structure impedance model $[43,48,49]$. The terminal resistance is simply given by

$$
R_{n}(L)_{\text {terminal }}=\frac{p_{\text {terminal }}}{Q_{n}(L)_{\text {terminal }}}
$$

Equation (9) allows us to update the terminal resistance at every step to match the physiological conditions of $P_{\text {terminal }}$. The terminal pressure is set in accordance with medical data $[49,50]$. These modifications to the $0 \mathrm{D}$ resistance model allow for the tree model to be extended to the terminal radius $(12 \mu \mathrm{m}$ in our model), where the pressure was set to $30 \mathrm{mmHg}$ (Fig. 4).

The special shape of the ICA, which does not branch until it enters the skull, was not included in this study as it lies outside of the region of interest. Therefore, in this study, the length and diameter of the ICA were determined on the basis of actual medical data.

Table 1 Diameter, length and ratio of vessel length to vessel radius $(\lambda)$ of each vessel in our peripheral network

\begin{tabular}{lllll}
\hline Blood vessel & Ave. diameter $(\mathbf{m m})$ & Min. diameter $(\mathbf{m m})$ & Length $(\mathbf{m m})$ & $\boldsymbol{\lambda}$ \\
\hline ICA (all mean) & 5.4834 & 4 & 117.3925 & 50.0154 \\
Large arteries & 6.5 & 2 & 200 & 61.5385 \\
Main artery branches & 2.4 & 1.2 & 100 & 83.3333 \\
Terminal artery branches & 1.2 & 0.1 & 10 & 16.6667 \\
Arterioles & 0.1 & 0.015 & 2 & 40 \\
Capillaries & 0.008 & 0.004 & 1 & 250 \\
\hline
\end{tabular}

The values for the diameter and length of the internal carotid artery (ICA) are mean values over the four 3D models, and the $\lambda$ value of each vessel is calculated from its length and half its averaged diameter 


\section{Modelling of non-Newtonian viscosity}

In large and medium arteries, blood behaves as a Newtonian fluid, and the viscosity. Blood has usually been treated as a Newtonian fluid in CFD studies of large and medium arteries. It does not exhibit a constant viscosity at all flow rates and is especially nonNewtonian in the microcirculatory system. Blood also exhibits non-Newtonian behaviour in small branches and capillaries, where the cells squeeze through and a cell-free skimming layer reduces the effective viscosity through the tube. To take into account non-Newtonian effects, the apparent viscosity is given as a function of both the diameter and the haematocrit $[47,51-53]$. First, the haematocrit is given by

$$
H c t=\left\{\begin{array}{cl}
0.45 & \text { if } d>300 \mu \mathrm{m} \\
0.45(0.196 \log d-0.117) & \text { if } d \leq 300 \mu \mathrm{m}
\end{array}\right.
$$

where $d$ is the vessel diameter.

When the vessel diameter becomes smaller than $10 \mu \mathrm{m}$, the apparent viscosity decreases in accordance with the Fåhraeus-Lindqvist effect. By the inverse FåhraeusLindqvist effect, the apparent viscosity increases when the vessel diameter is larger than a certain value. Equation (10) incorporates both effects.

The red line $(H c t=f(d))$ in Fig. 5 shows the behaviour of Hct in this study. The apparent viscosity is given in previous studies [54, 55]. According to Freitas [47], the haematocrit changes when the diameter $d$ becomes smaller than $300 \mu \mathrm{m}$. We used Eq. (11), which was proposed by Pries et al. [54, 55], to obtain the following relation for the changes in the viscosity $\mu_{\text {app }}$ associated with changes in vessel diameter $d$.

$$
\mu_{\text {app }}=\left[1+\left(\mu_{0.45}-1\right) \frac{(1-H c t)^{C d}}{(1-0.45)^{C d}}\left(\frac{d}{d-1.1}\right)^{2}\right]\left(\frac{d}{d-1.1}\right)^{2}
$$

where

$$
C d=(0.8+\exp [-0.075 d])\left(-1+\frac{1}{1+10^{-11} d^{12}}\right)+\frac{1}{1+10^{-11} d^{12}}
$$

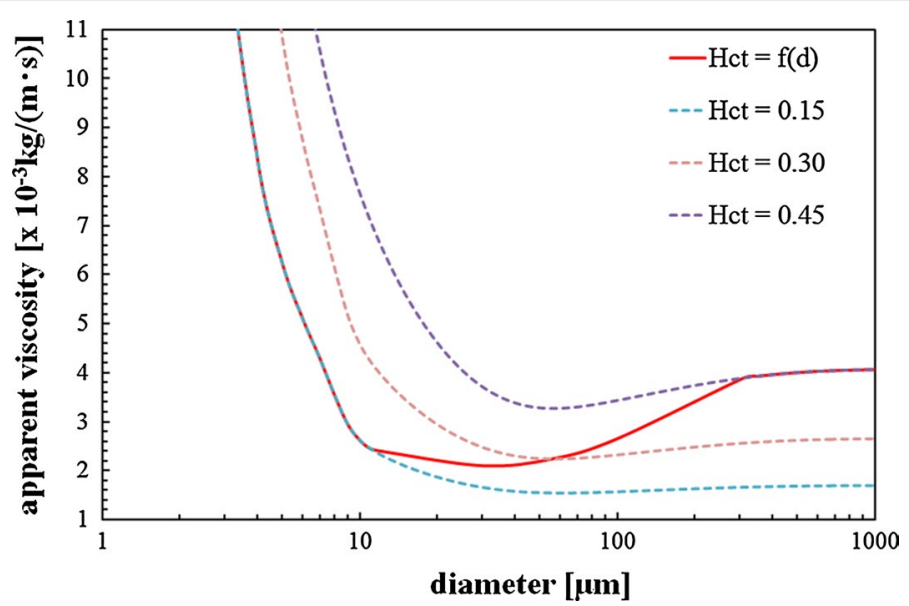

Fig. 5 Apparent viscosity with variable haematocrit 
and

$$
\mu_{0.45}=6 \exp [-0.085 d]+\mu_{\text {Newtonian }}-2.44 \exp \left[-0.06 d^{0.645}\right]
$$

When $\mu$ (the viscosity of the haematocrit) is $0.45, \mu_{\text {Newtonian }}$ is considered to be $4.06 \times 10^{-3}(\mathrm{~Pa} \cdot \mathrm{s})$.

\section{Doppler ultrasound measurement}

We defined the uniform flow velocity in a circumferential direction at the inlet to be the mean of the blood flow velocity for the cardiac cycle obtained by averaging four consecutive cardiac cycles. Blood flow measurement as a preoperative examination for intraarterial chemotherapy was conducted using a commercial ultrasound scanner (HDI 5000; ATL-Philips Medical Systems, WA). Doppler velocity measurement accuracy is $\pm 1 \%$ or $1 / 2$ pixel. Doppler velocity measurements are most accurate when the acoustic beam is aligned parallel with blood flow. All the data for this study were obtained by an experienced sonographer.

In each patient, B-mode ultrasound and colour Doppler imaging showed that each artery had no severe stenosis, obstructive lesions or meandering. Using colour Doppler imaging, velocities were determined at the centrelines of the CCA and STA. Pulsatile waveforms were obtained at the centreline of the distal CCA approximately $1-2 \mathrm{~cm}$ before the bifurcation without relevant flow disturbances, and at the centreline of the STA, velocities were determined at the front of the ear. The sample volume was set to be $2 / 3$ of the vessel lumen; the centre of the measurement site was in the long axis view; the angle correction was activated, and an angle of insonation of $60^{\circ}$ was maintained whenever possible.

\section{Results}

The simulations were conducted for 4 different types of vessel models (CCA, ICA, ECA and ECA branches). Each simulation took approximately $26 \mathrm{~h}$ (93,600 s) to complete.

Figure 6 shows the results for the pressure at each outlet in the $0 \mathrm{D}$ simulation. The pressures obtained with the present $0 \mathrm{D}$ resistance model were within the physiological range. The velocity magnitudes at the outlet of each vessel are summarized in Fig. 7. The velocity magnitude for the STA as given by both $0 \mathrm{D}$ resistance models is in agreement with the ultrasound data.

To verify the simulation results, the blood velocities in the STA in each simulation were compared with those obtained from ultrasound measurements, as shown in Table 2. The mean error was defined as the average of the errors of the four models compared with the actual value obtained from ultrasound measurements, and the error value was calculated using the above-mentioned three types of boundary conditions. The mean error in the STA velocities in the simulation was $48.02 \pm 22.66 \%, 5.32 \pm 4.52 \%$ and $5.21 \pm 0.78 \%$ for the zero-pressure outflow model, the conventional OD resistance model and the present OD resistance model, respectively (Fig. 8). The simulation based on the zero-pressure outflow boundary condition yielded the largest mean error of the three models, whereas both OD resistance models provided small mean errors. However, the standard deviation of the present $0 \mathrm{D}$ resistance model was smaller than that of the 


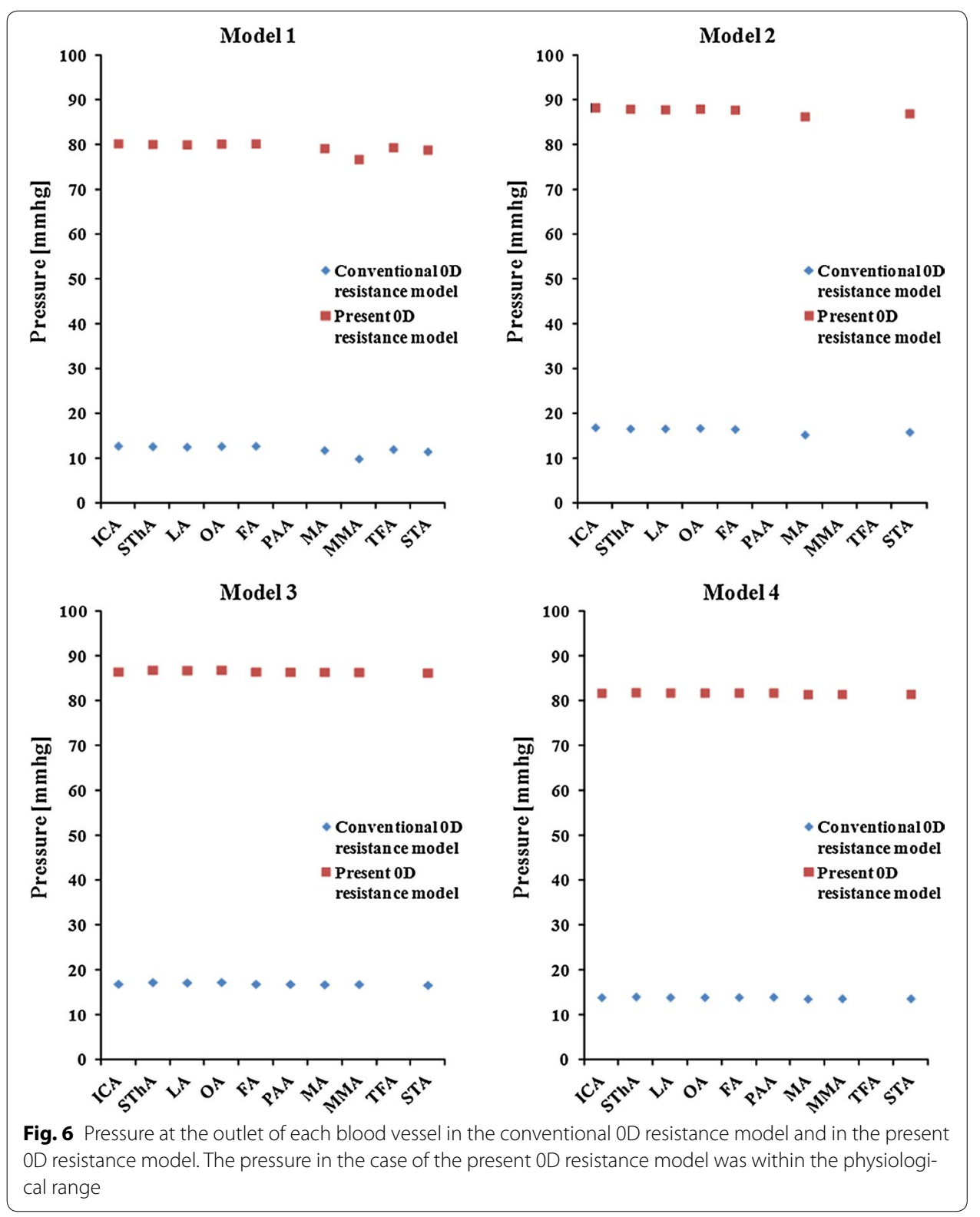

conventional 0D resistance model, which implies that simulation based on the present model is more precise.

\section{Discussion}

This study focused on head and neck haemodynamics for the treatment of oral cancer, especially in cases where intra-arterial chemotherapy is necessary. There have been few patient-specific blood flow simulations for oral cancer chemotherapy to date. Although Rhode et al. [56] previously reported the results of simulations of haemodynamic flow in head and neck cancer chemotherapy, where a patient-specific vessel model was created from CT images, branches of the ECA, such as the OA, MA and STA, were not modelled, and the peripheral vascular network was not considered. Our present OD 


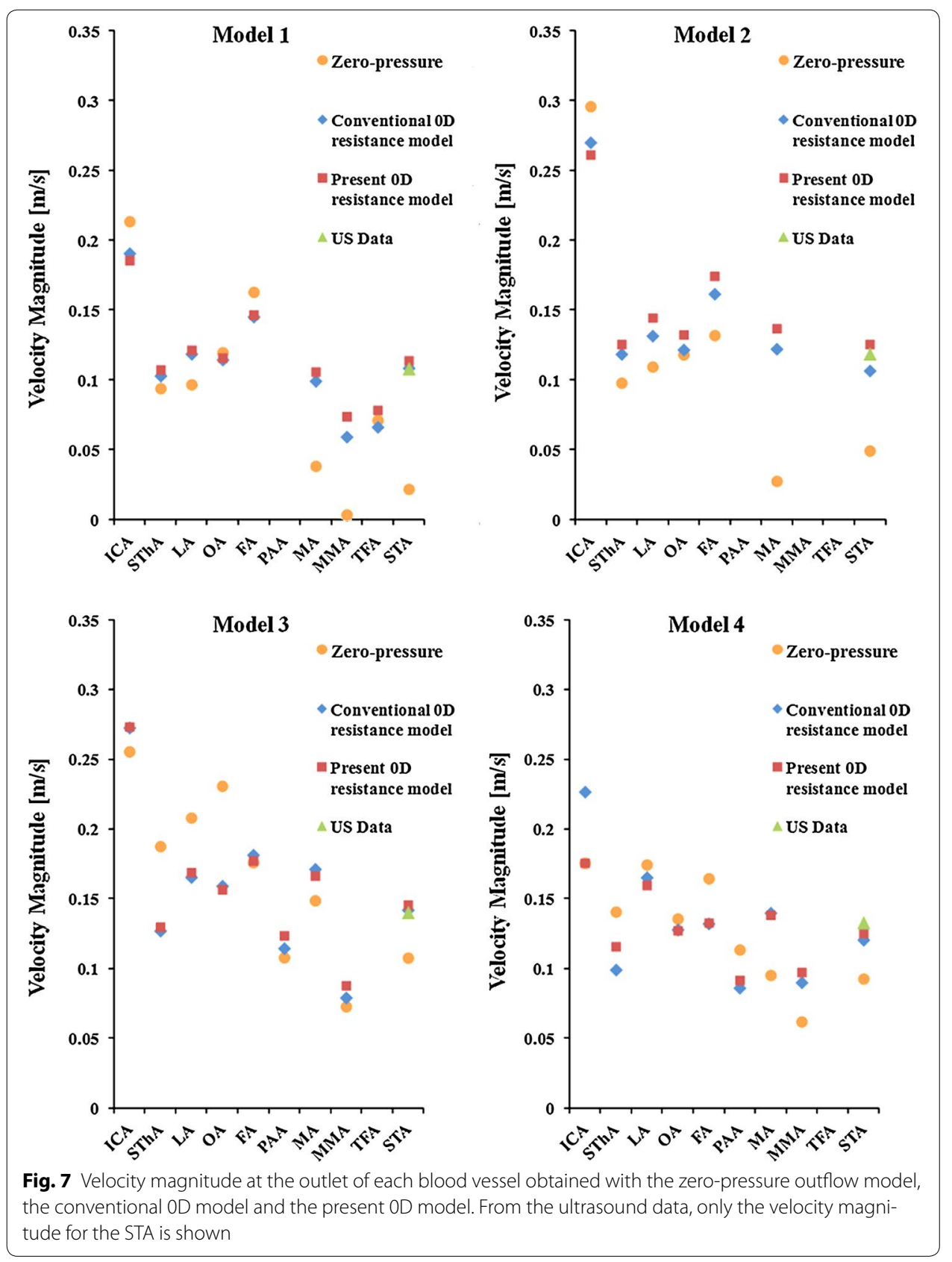

Table 2 Velocities for the superficial temporal artery obtained for the four models with each outlet boundary condition together with ultrasound measurement data

\begin{tabular}{lcclc}
\hline & Model $\mathbf{1}(\mathbf{c m} / \mathbf{s})$ & Model $\mathbf{2}(\mathbf{c m} / \mathbf{s})$ & Model 3 $(\mathbf{c m} / \mathbf{s})$ & Model 4 (cm/s) \\
\hline Zero-pressure outflow & 2.167 & 4.917 & 10.760 & 9.260 \\
Conventional OD resistance model & 10.834 & 10.643 & 14.196 & 12.049 \\
Present 0D resistance model & 11.368 & 12.538 & 14.565 & 12.525 \\
Ultrasound data & 10.796 & 11.854 & 14.016 & 13.304 \\
\hline
\end{tabular}




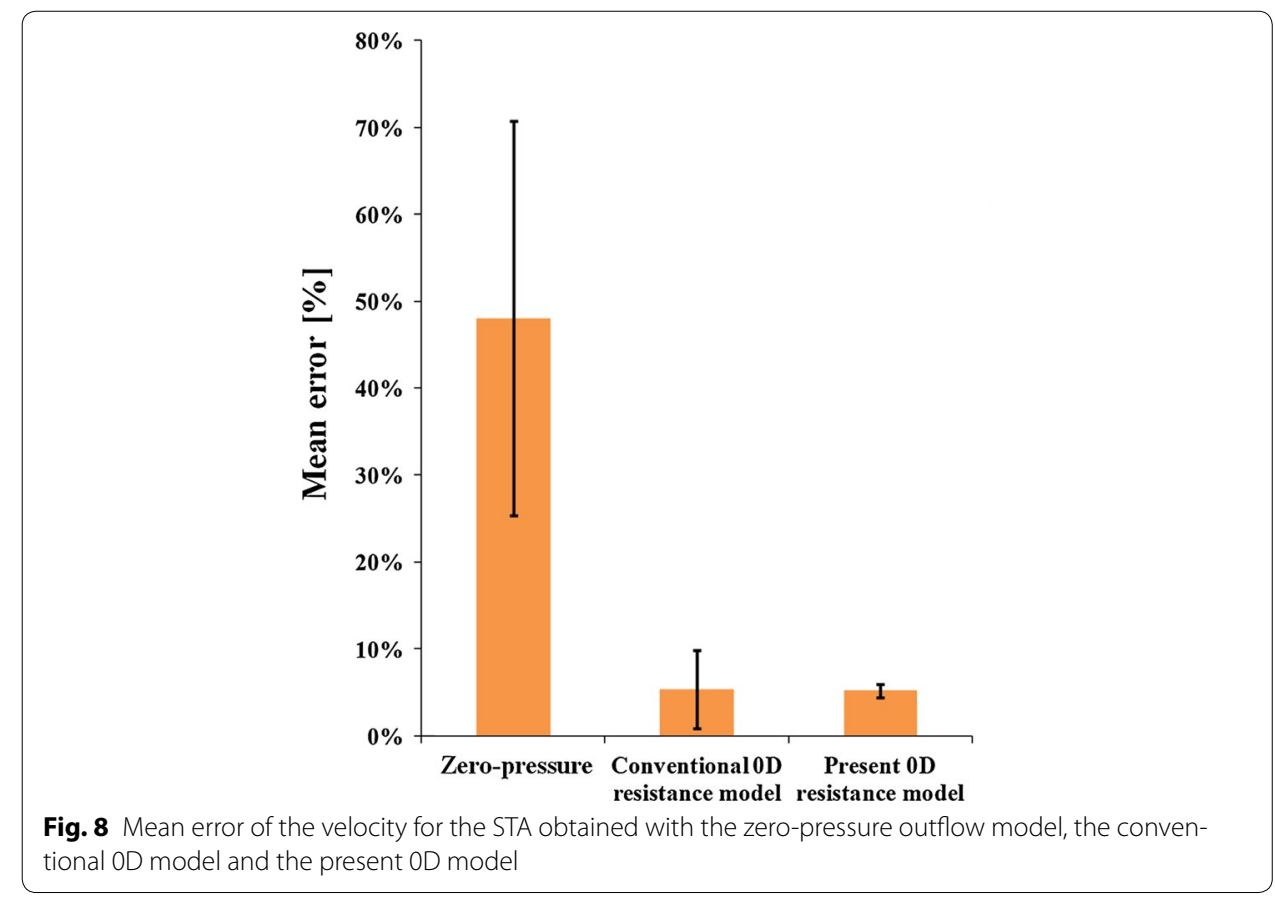

resistance model is clinically useful because it can provide an accurate estimation of the pressure and the flow distribution in vessels. The application of the present $0 \mathrm{D}$ resistance model as a boundary condition yields more realistic blood flow simulations the results of which can be used to achieve optimal tumour control with minimum accompanying side effects in oral cancer chemotherapy. In this paper, we consider a novel method for haemodynamic flow simulation applicable to intra-arterial chemotherapy for oral cancer.

The usefulness of our peripheral blood vessel model (0D resistance model) was verified using a patient-specific 3D model of the carotid arteries and branches together with ultrasound data of the CCA (the inlet of the blood flow) and the ECA (the outlet of the blood flow). The zero-pressure outflow boundary condition underestimated the STA velocity (compared with the results of ultrasound measurements) with a mean error of $48.02 \pm 22.66 \%$. Since the zero-pressure boundary condition considers none of the physiological effects of the peripheral vascular system, the flow distribution is determined by only the geometry of the analysis model constructed from medical images. On the other hand, the conventional and proposed OD resistance models consider the effects of the circulatory system. Thus, the mean error of the STA velocities was drastically reduced to $5.32 \pm 4.52 \%$ and $5.21 \pm 0.78 \%$, respectively. The results obtained with both $0 \mathrm{D}$ resistance models were in closer agreement with the measurement data than the results obtained using the free outflow boundary condition. Although the peripheral resistance in the present $0 \mathrm{D}$ resistance model tended to be greater than that in the conventional $0 \mathrm{D}$ resistance model, there was no significant difference in flow rate between the two 0D models. However, the present model correlated well with the experiment, yielding a standard deviation of $0.78 \%$ compared with $4.52 \%$ in the case of the conventional model. This improved precision may be because the present $0 \mathrm{D}$ resistance model determines $\lambda$ automatically and systematically based on anatomical knowledge, while the conventional OD model uses a fixed value $(\lambda=30)$. 
This study assumes a healthy blood vessel, but angiogenesis has been observed in the region of tumour progression. Therefore, it may be not reasonable to assume the parameters are the same for healthy blood vessels in the region with the tumour and further studies should be performed in the future.

\section{Conclusions}

We have presented some simple yet accurate outflow boundary conditions for conducting patient-specific blood simulations within a reasonable computation time for clinical applications. Since the region of interest in this study was the head and neck, the peripheral blood vessel network consists of mostly small blood vessels, which tend to be rigid. The outflow boundary conditions were designed based on the characteristics of the peripheral network by using the resistance of the blood vessel rather than the impedance, which combines compliance and resistance. To obtain an even more realistic simulation with the OD model, the parameter $\lambda$ was adjusted in accordance with anatomical knowledge.

The present $0 \mathrm{D}$ resistance model was used together with patient-specific 3D models of the carotid arteries to verify the simulation results, where ultrasound measurement results for the CCA and STA were used as reference data. The simulation based on the proposed OD resistance model boundary conditions correlated well with the ultrasound measurement data and was more precise than those based on either the zero-pressure outflow condition or the conventional OD resistance model. Furthermore, the pressure in the present $0 \mathrm{D}$ model was within the physiological range, in contrast to that of the conventional OD model.

Our OD resistance model is clinically useful because it can provide more realistic and accurate blood flow simulations, which may be helpful for achieving optimal tumour control with minimum accompanying side effects in oral cancer chemotherapy.

\section{Abbreviations}

OD: zero dimension; ECA: external carotid artery; ICA: internal carotid artery; 1D-0D: one dimensional-zero dimensional; 3D: three-dimensional; STA: superficial temporal artery; CT: computed tomography; CCA: common carotid artery; DICOM: digital imaging and communication in medicine; SThA: superior thyroid artery; LA: lingual artery; FA: facial artery; OA: occipital artery; MA: maxillary artery; PAA: posterior auricular artery; MMA: middle meningeal artery; TFA: transverse facial artery; FVM: finite volume method; MR: magnetic resonance; COE: Centers of Excellence.

\section{Authors' contributions}

YO conceived and designed the study, carried out the model parameterization and simulation studies, contributed in the data analysis and drafted the manuscript. MO participated in design of the study and coordinated the simulation activities and helped to draft the manuscript. TI carried out the clinical examination and participated in collecting the imaging data, helped in design of the study and participated in coordination and contributed in drafting the manuscript and revising it critically for important intellectual content. HK and YY participated in the work of simulation. AK participated in the work of model construction and provided technical supports. KM carried out the clinical examination and provided clinical supports. IT supervised the overall project, and conceived of the study. All authors read and approved the final manuscript.

\section{Author details}

${ }^{1}$ Department of Oral and Maxillofacial Surgery, Yokohama City University Graduate School of Medicine, 3-9 Fukuura, Kanazawa-ku, Yokohama 236-0004, Japan. ${ }^{2}$ Department of Interfaculty Initiative in Information Studies, The University of Tokyo, 7-3-1 Hongo, Bunkyo-ku, Tokyo 113-0033, Japan. ${ }^{3}$ Institute of Industrial Science, The University of Tokyo, 4-6-1 Komaba, Meguro-ku, Tokyo 153-8505, Japan.

\section{Acknowledgements}

This research was supported in part by the Global COE (Centers of Excellence) Program (Grant Number J07) and a Grantin-Aid for Scientific Research (B) (JSPS KAKENHI Grant Number 21390542), JSPS, Japan. 
Received: 26 September 2015 Accepted: 26 January 2016

Published online: 04 February 2016

\section{References}

1. Mitsudo K, Shigetomi T, Fujimoto Y, Nishiguchi H, Yamamoto N, Furue H, Ueda M, Itoh Y, Fuwa N, Tohnai I. Organ preservation with daily concurrent chemoradiotherapy using superselective intra-arterial infusion via a superficial temporal artery for T3 and T4 head and neck cancer. Int J Radiat Oncol Biol Phys. 2011;79:1428-35.

2. Cebral JR, Yim PJ, Löhner R, Soto O, Choyke PL. Blood flow modeling in carotid arteries with computational fluid dynamics and MR imaging. Acad Radiol. 2002;9:1286-99.

3. Keshavarz-Motamed Z, Garcia J, Kadem L. Fluid dynamics of coarctation of the aorta and effect of bicuspid aortic valve. PLoS One. 2013;8:e72394.

4. Kono K, Shintani A, Terada T. Hemodynamic effects of stent struts versus straightening of vessels in stent-assisted coil embolization for sidewall cerebral aneurysms. PLoS One. 2014;9:e108033.

5. Leng X, Scalzo F, I I HL, Johnson M, Fong AK, Fan FSY, Chen X, Soo YOY, Miao Z, Liu L, Feldmann E, Leung TWH, Liebeskind DS, Wong KS. Computational fluid dynamics modeling of symptomatic intracranial atherosclerosis may predict risk of stroke recurrence. PLoS One. 2014;9:1-8.

6. Lou Z, Yang WJ. A computer simulation of the non-Newtonian blood flow at the aortic bifurcation. J Biomech. 1993;26:37-49.

7. Milner JS, Moore JA, Rutt BK, Steinman DA. Hemodynamics of human carotid artery bifurcations: computational studies with models reconstructed from magnetic resonance imaging of normal subjects. J Vasc Surg. 1998;28:143-56.

8. Molla MM, Paul MC, Roditi G. LES of additive and non-additive pulsatile flows in a model arterial stenosis. Comput Methods Biomech Biomed Engin. 2010;13:105-20.

9. Paul MC, Larman A. Investigation of spiral blood flow in a model of arterial stenosis. Med Eng Phys. 2009;31:1195-203.

10. Paul MC, Mamun Molla M, Roditi G. Large-Eddy simulation of pulsatile blood flow. Med Eng Phys. 2009;31:153-9.

11. Paul MC, Molla MM. Investigation of physiological pulsatile flow in a model arterial stenosis using large-eddy and direct numerical simulations. Appl Math Model. 2012;36:4393-413.

12. Perktold K, Peter RO, Resch M, Langs G. Pulsatile non-Newtonian blood flow in three-dimensional carotid bifurcation models: a numerical study of flow phenomena under different bifurcation angles. J Biomed Eng. 1991;13:507-15.

13. Steinman DA, Thomas JB, Ladak HM, Milner JS, Rutt BK, Spence JD. Reconstruction of carotid bifurcation hemodynamics and wall thickness using computational fluid dynamics and MRI. Magn Reson Med. 2002;47:149-59.

14. Steinman DA, Hoi Y, Fahy P, Morris L, Walsh MT, Aristokleous N, Anayiotos AS, Papaharilaou Y, Arzani A, Shadden SC, Berg P, Janiga G, Bols J, Segers P, Bressloff NW, Cibis M, Gijsen FH, Cito S, Pallarés J, Browne LD, Costelloe JA, Lynch AG, Degroote J, Vierendeels J, Fu W, Qiao A, Hodis S, Kallmes DF, Kalsi H, Long Q, et al. Variability of computational fluid dynamics solutions for pressure and flow in a giant aneurysm: the ASME 2012 Summer Bioengineering Conference CFD Challenge. J Biomech Eng. 2013;135:021016.

15. Taylor CA, Hughes TJ, Zarins CK. Effect of exercise on hemodynamic conditions in the abdominal aorta. J Vasc Surg. 1999;29:1077-89.

16. Taylor CA, Fonte TA, Min JK. Computational fluid dynamics applied to cardiac computed tomography for noninvasive quantification of fractional flow reserve: scientific basis. J Am Coll Cardiol. 2013;61:2233-41.

17. Zhao SZ, Ariff B, Long Q, Hughes AD, Thom SA, Stanton AV, Xu XY. Inter-individual variations in wall shear stress and mechanical stress distributions at the carotid artery bifurcation of healthy humans. J Biomech. 2002;35:1367-77.

18. Takizawa K, Schjodt K, Puntel A, Kostov N, Tezduyar TE. Patient-specific computer modeling of blood flow in cerebral arteries with aneurysm and stent. Comput Mech. 2012;50:675-86.

19. Fishman EK, Kuszyk BS, Heath DG, Cabral B. Surgical planning for liver resection. Computer (Long Beach Calif). 1996;29:64-72.

20. Gijsen FJ, van de Vosse FN, Janssen JD. The influence of the non-Newtonian properties of blood on the flow in large arteries: steady flow in a carotid bifurcation model. J Biomech. 1999;32:601-8.

21. Nakajima S, Atsumi H, Bhalerao AH, Jolesz FA, Kikinis R, Yoshimine T, Moriarty TM, Stieg PE. Computer-assisted surgical planning for cerebrovascular neurosurgery. Neurosurgery. 1997;41:403-9.

22. Pahlavian SH, Yiallourou T, Tubbs RS, Bunck AC, Loth F, Goodin M, Raisee M, Martin BA. The impact of spinal cord nerve roots and denticulate ligaments on cerebrospinal fluid dynamics in the cervical spine. PLoS One. 2014;9:1-14.

23. Taylor CA, Hughes TJR, Zarins CK. Finite element modeling of blood flow in arteries. Comput Methods Appl Mech Eng. 1998;158:155-96.

24. Lee C-J, Uemiya N, Ishihara S, Zhang Y, Qian Y. A comparison of estimation methods for computational fluid dynamics outflow boundary conditions using patient-specific carotid artery. Proc Inst Mech Eng H. 2013;227:663-71.

25. Mei Y, Müller-eschner M, Chen D: The Hemodynamic Comparison of Different Carotid Artery Bifurcation Angles Based on Patient-Specific Models. In: Ma S, Jia L, Li X, Wang L, Zhou H, Sun X, editors. Life system modeling and simulation. Springer, Berlin, Heidelberg; 2014. p. 170-7.

26. Malvè M, Chandra S, García A, Mena A, Martínez MA, Finol EA, Doblaré M. Impedance-based outflow boundary conditions for human carotid haemodynamics. Comput Methods Biomech Biomed Engin. 2014;17:1248-60.

27. Sousa LC, Castro CF, António CC, Santos A, Santos R, Castro P, Azevedo E, Tavares JMRS. Haemodynamic conditions of patient-specific carotid bifurcation based on ultrasound imaging. Comput Methods Biomech Biomed Eng Imaging Vis. 2014;2(3):157-66.

28. Seo T. Numerical simulations of blood flow in arterial bifurcation models. Korea Aust Rheol J. 2013;25:153-61.

29. Piskin S, Serdar Celebi M. Analysis of the effects of different pulsatile inlet profiles on the hemodynamical properties of blood flow in patient specific carotid artery with stenosis. Comput Biol Med. 2013;43:717-28. 
30. Xu B, Zhong H, Duan S. Modeling of internal carotid artery aneurysm and blood flow simulation. Technol Heal Care. 2015;23:S43-8.

31. De Pater L, Van Den Berg JW. An electrical analogue of the entire human circulatory system. Med Electron Biol Eng. 1963;1964(2):161-6.

32. Westerhof N, Bosman F, De Vries CJ, Noordergraaf A. Analog studies of the human systemic arterial tree. J Biomech. 1969;2:121-43.

33. Noordergraaf A. Circulatory system dynamics. (Biophysics and Bioengineering Series, 1):V. 1. Academic Press Inc; 1978.

34. Mantero S, Pietrabissa R, Fumero R. The coronary bed and its role in the cardiovascular system: a review and an introductory single-branch model. J Biomed Eng. 1992;14:109-16.

35. Alastruey J, Parker K, Peiró J, Sherwin SJ. Lumped parameter outflow models for 1-D blood flow simulations : effect on pulse waves and parameter estimation. Commun Comput Phys. 2008;4:317-36.

36. Mirzaee MR, Ghasemalizadeh O, Firoozabadi B. Exact simulating of human arteries using lumped model and probing constriction in femoral and carotid arteries. Am J Appl Sci. 2009;6:834-42.

37. Liang FY, Takagi S, Himeno R, Liu H. Biomechanical characterization of ventricular-arterial coupling during aging: a multi-scale model study. J Biomech. 2009;42:692-704.

38. Caro CG, Pedley TJ, Schroter RC SW. The Mechanics of Circulation. J Fluid Mech. 90; 1979.

39. Zamir M. Nonsymmetrical bifurcations in arterial branching. J Gen Physiol. 1978;72:837-45.

40. Oshima M, Torii R, Tokuda S, Yamada S, Koizumi A. Patient-specific modeling and multi-scale blood simulation for computational hemodynamic study on the human cerebrovascular system. Curr Pharm Biotechnol. 2012;1-13:2153-65.

41. Uylings HBM. Optimization of diameters and bifurcation angles in lung and vascular tree structures. Bull Math Biol. 1977;39:509-20.

42. Rhodin JAG. Architecture of the vessel wall. In: Bohr DF, Somlyo AP, Sparks HV, editors. Handbook of physiology. Washington: American Physiological Soc; 1980. p. 1-31.

43. Olufsen MS, Peskin CS, Kim WY, Pedersen EM, Nadim A, Larsen J. Numerical simulation and experimental validation of blood flow in arteries with structured-tree outflow conditions. Ann Biomed Eng. 2000;28:1281-99.

44. Iberall AS. Anatomy and steady flow characteristics of the arterial system with an introduction to its pulsatile characteristics w. Math Biosci. 1967;1:375-95.

45. Suwa N, Niwa T, Fukasawa H, Sasaki Y. Estimation of intravascular blood pressure gradient by mathematical analysis of arterial casts. Tohoku J Exp Med. 1963;79:168-98.

46. Bassingthwaighte JB, van Beek JHGM. Lightning and the heart: fractal behavior in cardiac function. Proc IEEE Inst Electr Electron Eng. 2002;76:693-9.

47. Freitas RA. Nanomedicine, Volume I: Basic Capabilities. Landes Bioscience. 1999.

48. Steele BN, Olufsen MS, Taylor CA. Fractal network model for simulating abdominal and lower extremity blood flow during resting and exercise conditions. Comput Methods Biomech Biomed Engin. 2007;10:39-51.

49. Mahy IR, Tooke JE, Shore AC. Capillary pressure during and after incremental venous pressure elevation in man. J Physiol. 1995;485:213-9.

50. Rowell LB. Human Cardiovascular Control. USA: Oxford University Press; 1993.

51. Pries AR, Secomb TW, Gaehtgens P, Gross JF. Blood flow in microvascular networks experiments and simulation. Circ Res. 1990;67:826-34.

52. Barbee JH, Cokelet GR. The Fahraeus effect. Microvasc Res. 1971;3:6-16.

53. Barbee JH, Cokelet GR. Prediction of blood flow in tubes with diameters as small as 29 microns. Microvasc Res. 1971;3:17-21.

54. Pries AR, Neuhaus D, Gaehtgens P. Blood viscosity in tube flow: dependence on diameter and hematocrit. Am J Physiol. 1992;263:H1770-8.

55. Pries AR, Secomb TW, Gessner T, Sperandio MB, Gross JF, Gaehtgens P. Resistance to blood flow in microvessels in vivo. Circ Res. 1994;75:904-15.

56. Rhode S, Paul MC, Martens E, Campbell DF. Simulation of haemodynamic flow in head and neck cancer chemotherapy. Biomed Eng Online. 2011;10:104.

\section{Submit your next manuscript to BioMed Central and we will help you at every step:}

- We accept pre-submission inquiries

- Our selector tool helps you to find the most relevant journal

- We provide round the clock customer support

- Convenient online submission

- Thorough peer review

- Inclusion in PubMed and all major indexing services

- Maximum visibility for your research

Submit your manuscript at www.biomedcentral.com/submit 